\title{
BQS-Patientinnenbefragung für Frauen mit primärem Mammakarzinom
}

\author{
E. Schäfer ${ }^{1}$, N. Birkner ${ }^{1}$, F.J. Grothaus ${ }^{1}$, M. Dombrowski², S.
} Handstein $^{3}$, A. Lorenz-Wangard ${ }^{4}$, C. Mühr ${ }^{5}$, H. Schulte ${ }^{6}$, T. War$\mathrm{da}^{7}$, C. Veit ${ }^{1}$

Für Frauen mit Brustkrebs ist die aktive Beteiligung an der Entscheidung zur operativen Therapie ein wichtiger und wünschenswerter Bestandteil ihrer Versorgung [1]. Eine Partizipative Entscheidungsfindung (Shared Decision Making) setzt eine umfassende und qualifizierte Beratung der Patientinnen voraus, bei der sie angemessen über ihre Behandlungsoptionen, den Therapieverlauf und unterstützende Hilfsmaßnahmen informiert werden [2, 3]. Der neu entwickelte BQS-Mamma-Ca-Fragebogen zeigt Stärken und Schwächen der Qualität der präoperativen ärztlichen Beratungsgespräche auf. Gute Ergebnisse finden sich bei den BQS-Qualitätsindikatoren (QI): Behandlungsoptionen BET und Mastektomie (72,3\%), Voraussetzungen und Konsequenzen von BET und Mastektomie (88,3\%) sowie Empathie und Zeit für die Patientinnen (89,7\%). Verbesserungspotenzial zeigen die Indikatoren zu den Auswirkungen der Therapie auf das Frausein (45,2\%), Nennung professioneller Unterstützungsmöglichkeiten (45\%), Informationen zum Brustaufbau und äußerlich optische Angleichung (37,8\%) und zur Information, dass kein Zeitdruck für die OP besteht $(25,7 \%)$.

In den existierenden Verfahren der Qualitätssicherung in der Mammachirurgie bleiben Langzeitergebnisse aus Patientinnensicht (Patient Reported Outcome) trotz ihrer zentralen Bedeutung weitgehend unberücksichtigt. Diese können nur durch eine direkte Befragung der Patientinnen erhoben werden. Aus diesem Grund hat das BQS-Institut zusammen mit einer Projektgruppe ein Befragungsinstrument zur Erfassung patientinnenrelevanter Qualitätsaspekte der Brustkrebstherapie entwickelt. Diese Gruppe setzte sich zusammen aus Patientinnenvertreterinnen und medizinisch-pflegerischen Fachexperten der BQS-Fachgruppe Mammachirurgie.

Im Fokus dieser Qualitätssicherung steht das Modell der Partizipativen Entscheidungsfindung im präoperativen ärztlichen
Beratungsgespräch und hier insbesondere das aktive Informationsverhalten der Ärzte. Die vitale Bedeutung der auf der Basis der ärztlichen Beratung getroffenen Therapieentscheidungen erfordert eine umfassende Information der Patientinnen.

Zur Befragung der Patientinnen wurde ein Fragebogen (BQS-Mamma-Ca-Fragebogen) entwickelt und in 2 Pretests getestet und validiert. Dabei definierte die Projektgruppe aus den empirischen Ergebnissen 16 spezifische BQSQualitätsindikatoren. Die Ergebnisse des 2. Pretests werden in diesem Beitrag vorgestellt.

\section{Fragebogenentwicklung \\ $\nabla$}

Da kein Instrument existierte, das die Beratungsqualität als Benchmark messen konn- te, wurde ein Konzeptfragebogen für Patientinnen mit einem primären Mammakarzinom entwickelt. Da viele Patientinnen erst im Verlauf der Behandlung erkennen, ob sie alle notwendigen Informationen präoperativ erhalten haben, wurde die Befragung mit einem 6-monatigen Verzug durchgeführt. Der Retest nach weiteren 3 Monaten bestätigte die Stabilität der Ergebnisse.

Der erste Entwurf eines Fragensets wurde mittels Think-aloud-Interviews von Patientinnen getestet. Hierbei beantworteten die Patientinnen den Fragebogen, in dem sie die Fragen, ihre Antworten und ihre Gedanken hierzu laut äußerten, wodurch die Projektleiter Verständnisschwierigkeiten oder Missverständnisse erkennen konnten. Es entstand ein Fragebogen mit 172 Fragen. Dieser wurde im ersten Pretest von 206 Patientinnen aus 32 Kranken-häusern beantwortet.

Die Ergebnisse wurden faktorenanalytisch ausgewertet und der Fragebogen überarbeitet. Der revidierte Fragebogen mit 106 Fragen wurde in einem 2. Pretest von 401 Patientinnen aus 47 Krankenhäusern beantwortet.

Die Rücklaufquote lag bei $56 \%$ im ersten bzw. $59 \%$ im 2. Pretest (ohne Reminderverfahren). Das Alter der Patientinnen lag zwischen 26 und 86 Jahren (Md: 59; MW: 57,8) im ersten bzw. 24 und 87 Jahre (Md: 60; MW: 59,1) im 2. Pretest. Diese Daten sind vergleichbar mit der Altersverteilung in der Bundesauswertung 2009 des Leistungsbereichs Chirurgie bei Mammakarzinom (Md: 60; MW: 59,4).

Die Patientinnen der teilnehmenden Krankenhäuser wurden über die Befragung informiert und die unterschriebenen Einwilligungserklärungen an das BQS-Institut gesandt. Das BQS-Institut verschickte 6 Monate später den Fragebogen an die Patientinnen.

Die Krankenhäuser, denen die Teilnahme an diesen Pretests angeboten wurde, wurden durch eine Stichprobenziehung des BQS-Instituts ermittelt. Hierbei wurde darauf geachtet, dass sowohl Krankenhäuser aus allen Bundesländern wie auch mit unterschiedlich großen Fallzahlen teilnahmen. Auf diese Weise wurde sichergestellt, dass den statistischen Analysen eine Patientenstichprobe zugrunde gelegt wurde, die möglichst verschiedene Versorgungskonstellationen einbezog.

In beiden Pretests wurden Qualitätsdimensionen identifiziert, die ein sparsames Modell zur Darstellung des Beratungsgeschehens bilden und sich im besonderen Maße als Qualitätsindikatoren eignen. Auf 


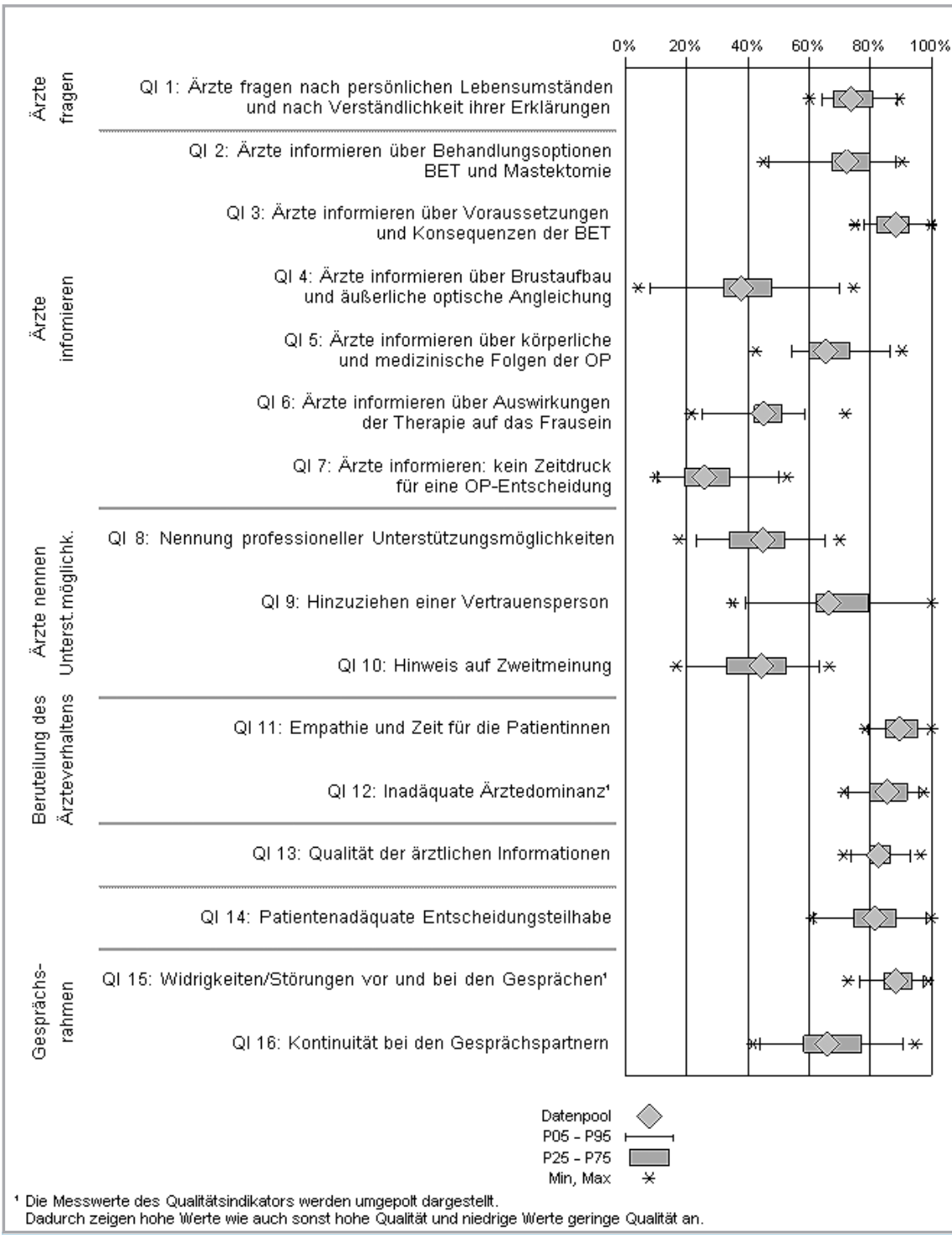

Abb. 1 Übersicht der Ergebnisse der 16 BQS-Qualitätsindikatoren.

Basis dieser Dimensionen hat die Projektgruppe 16 BQS-Qualitätsindikatoren definiert (vgl. $\triangle$ Abb. 1), die sich mit diesem Fragebogen messen lassen. Die Zahl der Fragen im endgültigen Fragebogen konnte auf insgesamt 86 reduziert werden.

Die Fragen beziehen sich auf Erfahrungen der Patientinnen, ob Informationen $\mathrm{zu}$ medizinischen und psychosozialen Aspekten vermittelt wurden, ob Nachfragen gestellt und die Patientinnen aktiv einbezogen wurden. Zusätzlich sind Fragen zu Globalbeurteilungen und Patientinnenmerkmale zu beantworten.

Die Qualitätsindikatoren des Fragebogens wurden bezüglich Validität, Reliabilität und Diskriminationsfähigkeit von den Fachexperten als gut bis sehr gut bewertet, so dass sich der Fragebogen für den Krankenhausvergleich eignet.

\section{Auswertungen für die Krankenhäuser $\nabla$} den und aussagekräftigen Ergebnisbericht, in dem die eigenen Ergebnisse den Gesamtergebnissen aller Teilnehmer gegenübergestellt sind. Das Auswertungskonzept reicht von Übersichten über die 16 BQS-Qualitätsindikatoren bis zu den detaillierten Einzelfragen. Die Ergebnisse der einzelnen Qualitätsindikatoren werden in Benchmark-Diagrammen dargestellt. Die Ergebnisse aller Qualitätsindikatoren werden mit Hilfe eines Punkteschemas so dargestellt und berechnet, dass hohe Werte hohe Qualität und niedrige Werte geringe Qualität anzeigen.

\section{Ergebnisse des 2. Pretests $\nabla$}

Die Antworten der befragten Patientinnen des 2. Pretests erlauben relevante
Die Krankenhäuser erhielten einen umfassen-
Trendaussagen zur Qualität der Beratung von Patientinnen mit einem primärem Mammakarzinom. Diese Ergebnisse werden im Folgenden dargestellt.

Die Verteilung der Krankenhausergebnisse aller 16 BQS-Qualitätsindikatoren (QI) ist in -Abb. 1 dargestellt. Es zeigen sich große Differenzen zwischen den Qualitätsindikatoren.

Auffällig sind die niedrigen Gesamtergebnisse der QI 4, 6, 7, 8 und 10 aus den Themenblöcken „Ärzte informieren“ und „Ärzte nennen Unterstützungsmöglichkeiten“ im Vergleich zu den Ergebnissen der übrigen QI. Im Einzelnen wurden folgende Ergebnisse erreicht:

- QI 4 „Ärzte informieren über Brustaufbau und äußerliche optische Angleichung“: $37,8 \%$

- QI 6 „Ärzte informieren über Auswirkungen der Therapie auf das Frausein“: $45,2 \%$

- QI 7 „Ärzte informieren: Kein Zeitdruck für eine OP-Entscheidung“: 25,7\%

- QI 8 „Nennung professioneller Unterstützungsmöglichkeiten“: 45,0\%

- QI 10 „Hinweis auf Zweitmeinung“: 44,6\%.

Die im Durchschnitt erreichten Werte liegen hier unter $50 \%$ mit großer Streuung der einzelnen Krankenhausergebnisse. Die Ergebnisse von QI 4 und QI 7 liegen sogar unter $40 \%$ bzw. $30 \%$ und zeigen somit auf, dass die Patientinnen hier große Mängel empfanden.

Mittlere Ergebnisse wurden bei den QI 5, 9 und 16 erreicht:

- QI 5 „Ärzte informieren über körperliche und medizinische Folgen der OP“: $65,4 \%$

> QI 9 „Hinzuziehen einer Vertrauensperson“: 66,6\%

- QI 16 „Kontinuität bei den Gesprächspartnern“: 66,1\%

Demgegenüber erreichten die Krankenhäuser gute bis sehr gute Ergebnisse bei den Qualitätsindikatoren zu den Themenblöcken „Ärzte fragen“, „Ärzte Informieren“ (z. T.), „Beurteilung des Ärzteverhaltens“, „Beurteilung der Informationen“, „Entscheidungsmitwirkung“ und „Gesprächsrahmen“. Im Einzelnen:

- QI 1 „Ärzte fragen nach persönlichen Lebensumständen und nach Verständlichkeit ihrer Erklärungen“: 73,6\%

- QI 2 „Ärzte informieren über Behandlungsoptionen BET und Mastektomie“: $72,3 \%$

- QI 3 „Ärzte informieren über Voraussetzungen und Konsequenzen der BET“: $88,3 \%$ 
- QI 11 „Empathie und Zeit für die Patientinnen“: 89,7\%

> QI 12 „Inadäquate Ärztedominanz“: 85,6\%*

\ QI 13 „Qualität der ärztlichen Informationen": 82,6\%

- QI 14 „Patientenadäquate Entscheidungsteilhabe“: $81,4 \%$

- QI 15 „Widrigkeiten/Störungen bei den Gesprächen“: 88,6\%*

Die Gesamtwerte liegen bei diesen Indikatoren zwischen 72,3\% und 89,7\% mit vergleichsweise geringer Streuung der einzelnen Krankenhausergebnisse.

Das Gesamtergebnis der einzelnen Qualitätsindikatoren setzt sich aus den Ergebnissen mehrerer Einzelfragen zusammen.

-Abb. 2 stellt das Ergebnis des QI 2 „Ärzte informieren über die Behandlungsoptionen BET und Mastektomie“ für 32 Krankenhäuser mit mindestens 5 zurückgesandten Patientinnenfragebögen dar, bei denen $352 \mathrm{~Pa}$ tientinnen eine Antwort gegeben haben. Mit dem Gesamtergebnis von 72,3\% liegt das Ergebnis im oberen Bereich, zeigt jedoch noch Verbesserungspotenzial. Die Betrachtung des Benchmarks bietet eine übersichtliche Einordnung der Ergebnisse der Krankenhäuser im Gesamtvergleich. Die Ergebnisse der zu diesem Indikator gehörenden Einzelfragen zeigen niedrigere Ergebnisse in der Informationsvermittlung, dass sich die Patientin zwischen 2 möglichen Operationsverfahren entscheiden kann (50,5\% positive Antworten) und der Aufklärung über die unterschiedlichen Konsequenzen dieser Operationsverfahren (59,7\% positive Antworten). Die positiven Antworten setzen sich aus den Antwortkategorien „trifft voll und ganz zu“ und „trifft eher zu“ zusammen.

Im QI 5 wird die Information über die körperlichen und medizinischen Folgen der OP durch 5 Items abgebildet. Ein gutes Ergebnis findet sich bei der Frage zur Aufklärung über mögliche Folgen der Operation (73,2\%). Etwas niedrigere Ergebnisse erzielen die Fragen, ob über mögliche Begleiterscheinungen der Bestrahlung informiert $(58,2 \%)$ und ob der Hinweis auf möglicherweise erforderliche weitere Operationen (55,6\%) gegeben wurde. Zudem wird die Frage, ob darauf hingewiesen wurde, dass das Gefühl in der operierten Brust verändert sein kann, nur von 54,3\% der Patientinnen mit „trifft voll und ganz zu“ und „trifft

\footnotetext{
* Die Messwerte des Qualitätsindikators werden ungepolt dargestellt. Dadurch zeigen hohe Werte Hohe (gute) Qualität an.
}

eher zu“ beantwortet. Die Information, dass Angleichungsoperationen an der anderen Brust später durchgeführt werden können, hat mit 32,4\% positiver Antworten den niedrigsten Wert dieser 5 Fragen ( $\bullet$ Abb. 3 ).

\section{Diskussion der Ergebnisse $\nabla$}

Ziel dieses Pretests war nicht, belastbare Ergebnisse zu liefern, sondern den Fragebogen und die Auswertung zu einem Reifegrad zu entwickeln, dass sie für zukünftige Befragungen validiert ist. Diese Befragung diente in erster Linie der Entwicklung des Fragebogens und Auswertungskonzepts und Erprobung der technischen Durchführung der Befragung. Es handelt sich daher nicht um eine epidemiologische Studie im engeren Sinne mit dem Ziel der Gewinnung repräsentativer Ergebnisse.

Hier sollen die Trends des Pretest dargestellt werden, ohne dass Anspruch auf Repräsentativität erhoben wird. Die Patientinnen im 2. Pretest berichten über ihre Erfahrungen in 47 Einrichtungen aus 14 Bundesländern mit einem Fallzahlvolumen von 77 bis 756 behandelten Patientinnen im Jahr 2009 (MW =259). Der Anteil Brust- zentren (22 KH) versus Nicht-Brustzentren $(25 \mathrm{KH})$ war relativ gleich verteilt.

Die Ergebnisse zeigen Stärken und Schwächen in den ärztlichen Beratungsgesprächen der Patienten auf, die geantwortet haben. Über die Erfahrungen der nicht-antwortenden Patientinnen liegen keine Erkenntnisse vor und sind nur schwer zu erlangen. Als Gründe für eine Nicht-Teilnahme wurden die Länge des damaligen Fragebogenentwurfs mit 106 Fragen und der Wunsch, sich mit diesem Thema nicht mehr beschäftigen zu wollen, angegeben.

Die ersten Ergebnisse zeigen, dass die Ärztinnen und Ärzte den Patientinnen in den Beratungsgesprächen insgesamt zugewandt und empathisch gegenübertreten (QI 11, 14 und 15). Sie geben verständliche, angemessene und umfassende Informationen (QI 1, 2, 3 und 13) und werden nicht als inadäquat dominant (QI 12) empfunden. Allerdings scheint die Information auf medizinische Sachverhalte und organisatorische Fragen fokussiert zu sein. Die körperlichen, medizinischen und psychischen Konsequenzen der Erkrankung und Therapie scheinen weniger im Aufmerksamkeitsfokus der Ärzte zu stehen (QI 5, 6, 8, 9, 10 und 16).

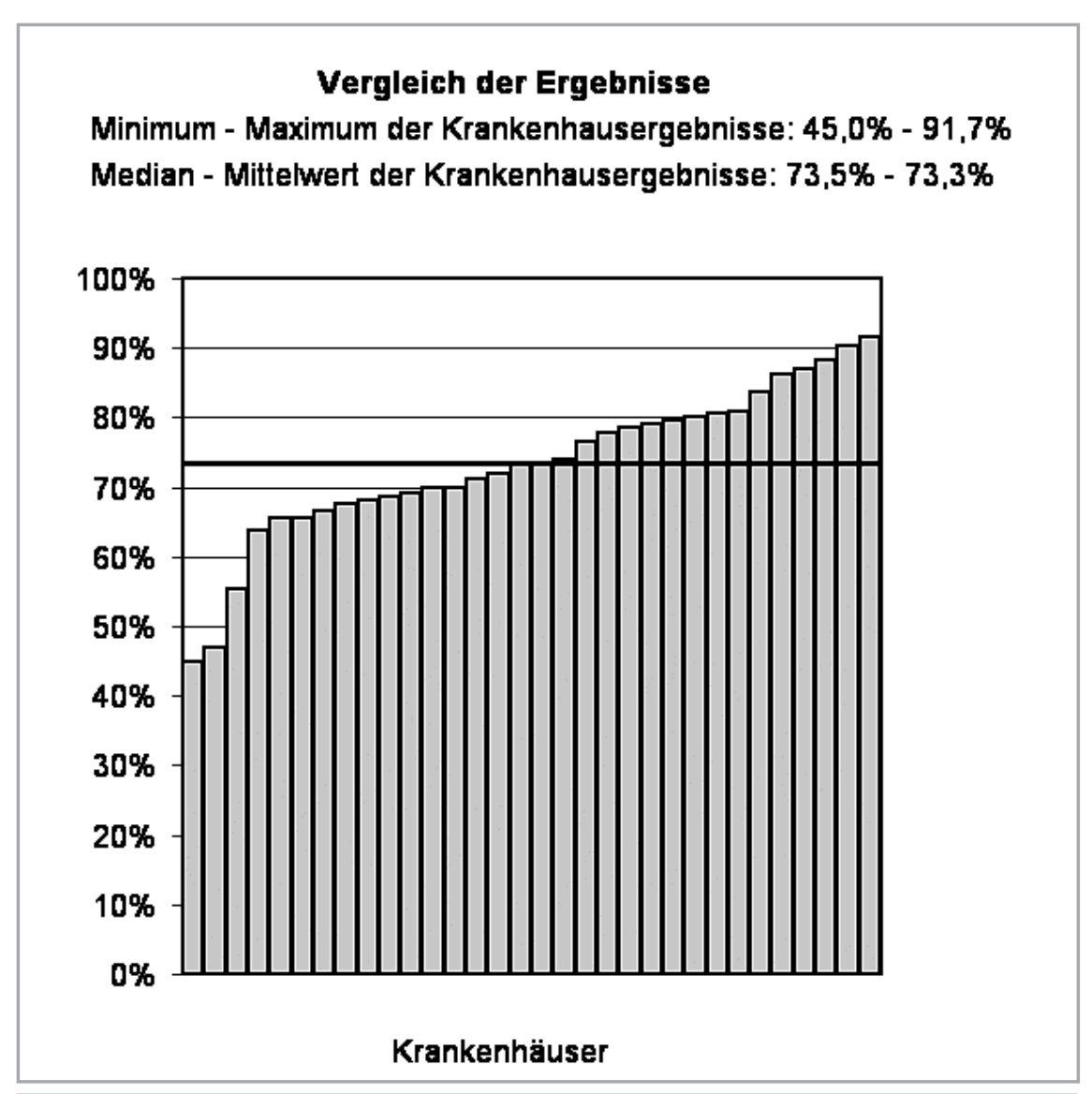

Abb. 2 Krankenhaus-Ergebnisse QI 2: „Ärzte informieren über Behandlungsoptionen BET und Mastektomie“. 
Zu den Aspekten, die von diesen Patientinnen weniger gehört oder erinnert wurden, gehört auch die Information über die grundsätzliche Möglichkeit einer (späteren) Rekonstruktion oder prothetischen Versorgung bei einer Entscheidung zur Mastektomie. Der QI 4 „Ärzte informieren über Brustaufbau und äußerliche optische Angleichung“ hat den zweitniedrigsten Wert aller Qualitätsindikatoren. Die Projektgruppenmitglieder und insbesondere die Patientinnenvertreterinnen messen einer solchen frühzeitig gegebenen Information eine sehr hohe Bedeutung bei, weil hierdurch die Wahlmöglichkeit zur BET oder Mastektomie entscheidend beeinflusst werden kann.

Obwohl in der Leitlinie Fraueninformation wie auch in der S3-Leitlinie [2, 3] ausdrücklich betont wird, dass Brustkrebs kein Notfall sei, wurde der aktive Hinweis durch Ärzte, dass kein Zeitdruck für eine schnelle Operation besteht (QI 7) nur in 26\% der Fälle mit „trifft voll und ganz zu“ und „trifft eher zu“ beantwortet. Die dazugehörigen Fragen holen Informationen ein, ob die Ärzte informierten, dass es nicht nötig sei, die OP möglichst schnell durchzuführen (31\% positive Antworten) und ob die Ärzte empfahlen, sich mehr Zeit für die Entscheidung zu nehmen (21\% positive Antworten).

Die Ergebnisse des Pretests zeigen auch, dass bei relativ gut bewerteten Qualitätsindikatoren einzelne Aspekte in den zugehörigen Einzelfrage teilweise erhebliches Verbesserungspotenzial aufweisen.

Inwieweit diese Ergebnisse als repräsentative Aussagen angesehen werden können, bleibt aufgrund des Pilotcharakters dieser Befragung weiteren bundesweiten Befragungen mit einer höheren Anzahl an Patientinnen vorbehalten. Unklar ist ebenfalls, ob die nicht antwortenden Patientinnen die Ergebnisse maßgeblich verändert hätten. Für eine Abbildung der Versorgungssituation in Deutschland und das Aufzeigen potenzieller Verbesserungsoptionen werden daher weitere Befragungen durchzuführen sein.

\section{Zusammenfassung und Ausblick} $\nabla$

Die im 2. Pretest befragten Patientinnen vermissen bei der Beratung durch die behandelnden Ärzte vor allem ein Gespräch über ihre Gesamtsituation nach der Therapie und die Bedürfnisse, die über die medizinischen Kernfragen hinausgehen. Hier besteht aus Ihrer Sicht der größte Verbesserungsbedarf in der präoperativen Beratung. Für die Gewinnung repräsentativer Ergeb-

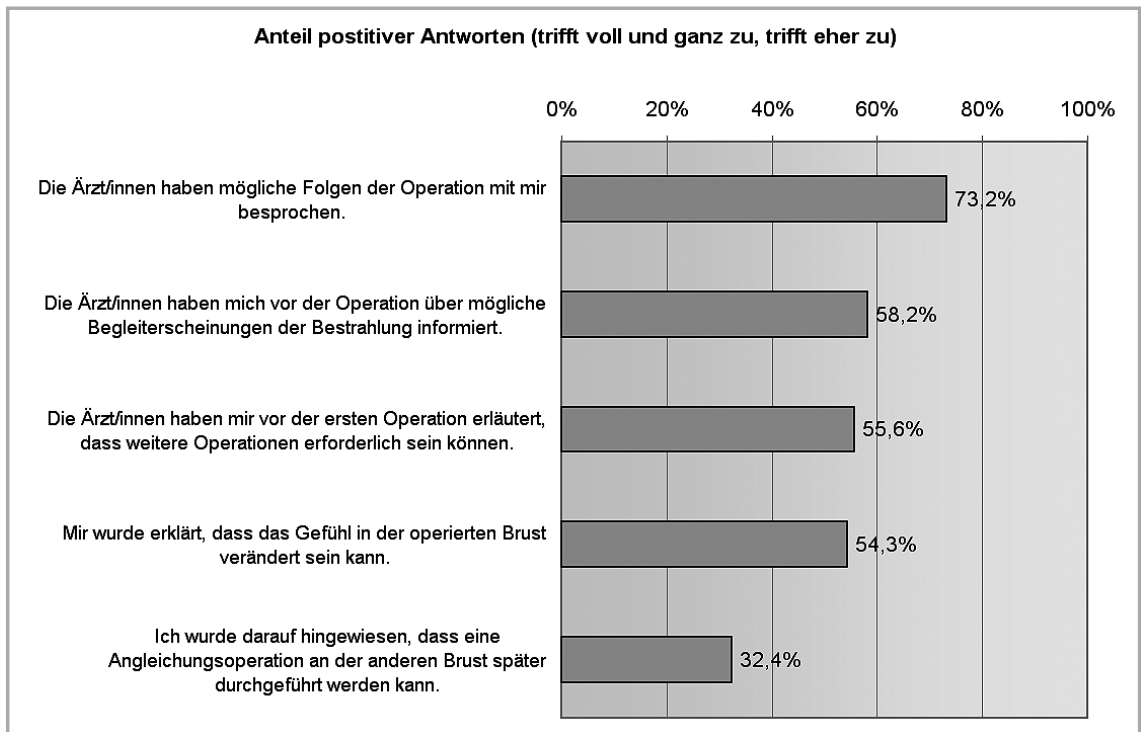

Abb. 3 Ergebnisse der Einzelfragen des QI 5: Ärzte informieren über körperliche und medizinische Folgen der OP.

nisse ist eine bundesweite Befragung mit einer größeren Anzahl an Patientinnen erforderlich, die das BQS-Institut interessierten Krankenhäusern ab Januar 2011 anbietet.

Das Verfahren hat durch die wissenschaftliche Entwicklung und Evaluation in den Pretests seine Validität und Praxistauglichkeit für die interne Qualitätssicherung gezeigt. Es hat gleichzeitig erste Hinweise auf Verbesserungsbedarf in der Versorgungsqualität von Patientinnen mit primärem Brustkrebs transparent gemacht. Im Zeitverlauf können Veränderungen in der Versorgungsqualität dargestellt werden. Ebenso ist es möglich zukünftig die Ergebnisse stratifiziert nach Größe und Ausrichtung der Einrichtungen, Brustzentren etc. auszuwerten.

Die Abfrage von Patientinnenerfahrungen (Patient Experiences) erlaubt dabei eine sehr viel differenziertere Darstellung der Verbesserungsmöglichkeiten als Zufriedenheitsabfragen. Die Entwicklung eines schlanken Erfassungsinstruments mit spezifischen Fragen für die besondere Behandlungssituation, erlaubt, fundierte Rückschlüsse auf die Qualität der Beratung von betroffenen Frauen.

Teilnehmende Krankenhäuser bekommen durch die Ergebnisse aufgezeigt, in welchen Bereichen bereits eine gute Qualität erreicht ist und wo Verbesserungen notwendig erscheinen. Zudem können diese Einrichtungen ihre Ergebnisse durch Benchmark-Grafiken mit anderen Krankenhäusern vergleichen. Die Krankenhäuser haben so die Möglichkeit, eine genaue Analyse ihrer Stärken und Schwächen vorzunehmen. Sie können konkrete Handlungsempfeh- lungen für ein gelungenes und strukturiertes Arzt-Patienten-Gespräch ableiten und diese durch entsprechende Schulung der Mitarbeiter umsetzen.

Literatur

1 Vodermaier A, Caspari C, Köhm J et al. Partizipative Entscheidungsfindung bei Patientinnen mit neu diagnostiziertem Brustkrebs - Überblick und Ergebnisse des Modellprojekts. In: Härter M, Loh A, Spies C (Hrsg.). Gemeinsam entscheiden - erfolgreich behandelt. Neue Wege für Ärzte und Patienten im Gesundheitswesen. Köln: Deutscher Ärzte-Verlag, 2005: 213-223

2 Albert US, Schulz KD, Alt D et al. Methodische Entwicklung und Anwendung der Leitlinie Fraueninformation, 2003. http://www. senologie.org/download/pdf/llfraueninformation.pdf (Recherche-datum: 11.10.2010)

3 Kreienberg R, Kopp I, Albert U et al. Interdisziplinäre S3-Leitlinie für die Diagnostik, Therapie und Nachsorge des Mammakarzinoms. In: Deutsche Krebsgesellschaft e.V. Informationszentrum für Standards in der Onkologie (ISTO) (Hrsg.). Berlin: Zuckschwerdt Verlag, 2008

Institutsangaben:

1 BQS Institut für Qualität und Patientensicherheit

2 Ev. Waldkrankenhaus Spandau

3 Städtisches Klinikum Görlitz

4 Vertreter der GKV und der PKV, Koblenz

5 Deutsche Arbeitsgemeinschaft Selbsthilfegruppen e.V., Berlin

6 Frauenselbsthilfe nach Krebs e.V., Bonn 7 Klinikum Minden

Korrespondenzautorin:

Elke Schäfer

$B Q S$ Institut für Qualität und Patientensicherheit

E-Mail: e.schaefer@bqs-institut.de

Internet: www.bqs-institut.de 